\title{
Extension of the characteristic equation to absorption chillers with adiabatic absorbers
}

\author{
G. Gutiérrez-Urueta ，P. Rodríguez , F. Ziegler , A. Lecuona , M.C. Rodríguez-Hidalgo
}

\begin{abstract}
A B S T R A C T
Various researchers have developed models of conventional $\mathrm{H}_{2} \mathrm{O}-\mathrm{LiBr}$ absorption machines with the aim of predicting their performance. In this paper, the methodology of characteristic equations developed by Hellmann et al. (1998) is applied. This model is able to represent the capacity of single effect absorption chillers and heat pumps by means of simple algebraic equations. An extended characteristic equation based on a characteristic temperature difference has been obtained, considering the facility features. As a result, it is concluded that for adiabatic absorbers a subcooling temperature must be specified. The effect of evaporator overflow has been characterized. Its influence on cooling capacity has been included in the extended characteristic equation. Taking into account the particular design and operation features, a good agreement between experimental performance data and those obtained through the extended characteristic equation has been achieved at offdesign operation. This allows its use for simulation and control purposes.
\end{abstract}

\section{Extension d'une équation caractéristique aux refroidisseurs à absorption munis d'absorbeurs adiabatiques}

Mots clés : Absorption ; Bromure de lithium ; Différence de température ; Eau glacée ; Conditionnement d'air

\section{Introduction}

Today's research about absorption systems includes the development of new machines, models to analyze and/or predict the performance of the chiller, and technologies for improving the heat and mass transfer processes. In this respect, adiabatic absorption is being investigated with the aim of developing compactness and efficiency. It consists on dispersing the weak solution inside an adiabatic chamber, extracting the absorption heat downstream using a compact heat exchanger. As a result, the heat and mass transfer processes are separated into two different apparatuses (Fig. 1).

Experimental and theoretical studies concerning adiabatic absorption include the works performed by Ryan (1994), Ryan et al. (1995), Summerer et al. (1997); among others. Venegas et al. $(2004,2005)$ and Arzoz et al. (2005) offer a summary 


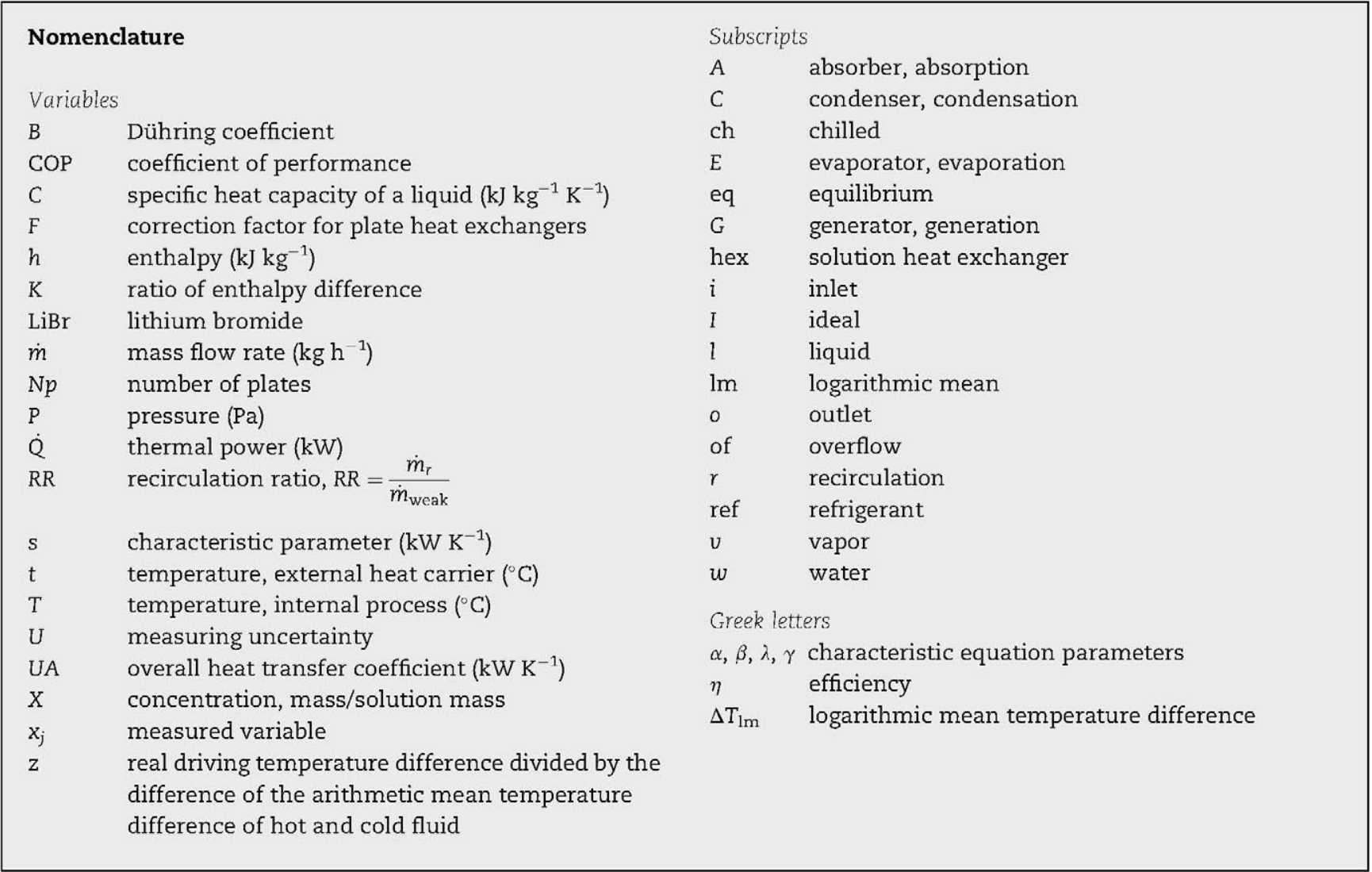

about those works. The literature review about recent publications in the field of adiabatic absorption using aqueous $\mathrm{LiBr}$ or lithium-based liquid absorbents is presented by GutiérrezUrueta et al. (2011). This includes the works reported by Warnakulasuriya and Worek (2006), Elperin et al. (2007) and Wang et al. (2007).

In another field of investigations, various researchers have developed models of conventional $\mathrm{LiBr}-\mathrm{H}_{2} \mathrm{O}$ absorption machines with the aim of predicting their performance. The effect of several operating variables or conditions on the

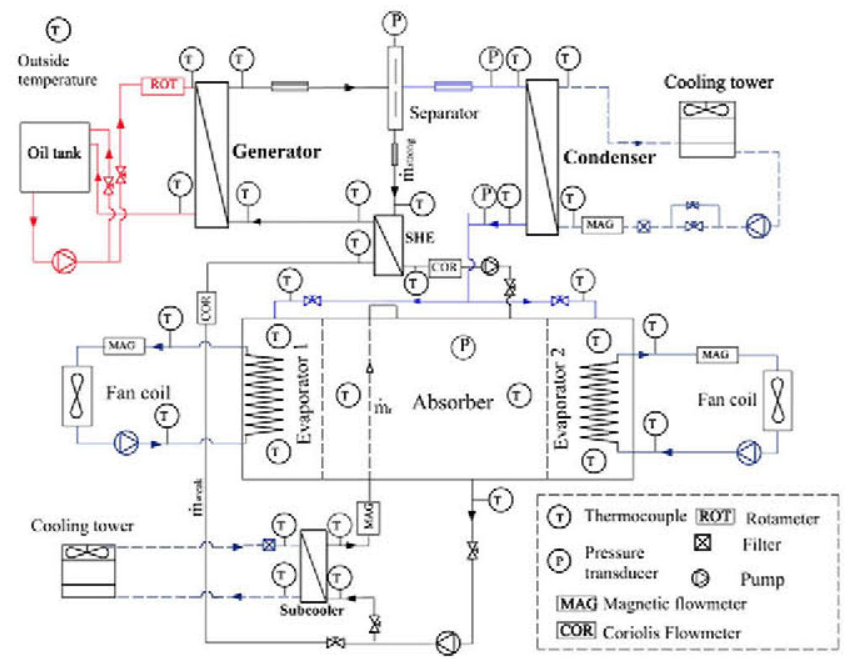

Fig. 1 - Flow diagram of the test rig, showing sensor positions. functioning of the absorption refrigeration system is a common objective in the papers by Hellmann et al. (1998), Joudi and Lafta (2001), Florides et al. (2003), among others. We want to use the model developed by Hellmann et al. (1998), which is able to represent both the capacity and the COP of single effect absorption chillers and heat pumps by means of simple algebraic equations. These equations are expressed as a function of the so called characteristic temperature difference, which depends on the average temperature of the external heat carrier fluids, and a parameter representing the slope of an average solution isostere in the Dühring chart, $B$ :

$\dot{\mathrm{Q}}_{E}=s \cdot \Delta \Delta \mathrm{t}-\alpha \cdot \dot{\mathrm{Q}}_{\text {loss }}$

With

$\Delta \Delta t:=t_{G}-t_{A}-B \cdot\left(t_{C}-t_{E}\right)$

Based on the mentioned work (Hellmann et al., 1998), cooling capacity is analyzed in this paper in terms of the characteristic temperature difference $\Delta \Delta$ t. Previous works (Albers and Ziegler, 2005 and Kühn and Ziegler, 2005) illustrate the use of $\Delta \Delta t$ to analyze and predict the offdesign performance of chillers with a diabatic absorber, meaning simultaneous absorption and cooling. In this paper, an extended version of the characteristic equation model has been developed and adapted to the features of the facility shown in Fig. 1. The adjustment of both the original and extended model to the data is presented for such particular equipment configuration that highlights the extended part. 


\section{Experimental setup}

Fig. 1 shows a diagram of the experimental setup. It consists of a single effect absorption cooling system, which incorporates plate heat exchangers (generator, condenser, subcooler and solution heat exchanger), two fin-coiled evaporators and the adiabatic absorber as main components. The evaporated refrigerant is absorbed by a flow of sub-cooled solution that is appropriately distributed inside the absorption chamber. The absorption heat is stored in the resulting solution and subsequently removed in a plate heat exchanger (subcooler). Both the solution coming from the generator $\dot{m}_{\text {strong }}$ and the recirculated solution $\dot{m}_{r}$ flow inside the absorber forming droplets or fan sheets. The experimental setup configuration, the data acquisition system, the experimental uncertainty analysis and the experimental procedure were described in detail in other publication (Gutiérrez-Urueta et al., 2011). Table 1 shows the characteristics of all sensors used in the experimental facility.

\section{Operational parameters}

In the following, the operational parameters used in the subsequent sections are defined.

The cooling capacity of the two evaporators at each side of the absorber, calculated through an energy balance on the external flow (Fig. 2) is:

$\dot{\mathrm{Q}}_{\mathrm{chw}}=\dot{\mathrm{m}}_{\mathrm{chw}} \mathrm{C}_{w}\left(\mathrm{t}_{\mathrm{chw}, \mathrm{i}}-\mathrm{t}_{\mathrm{chw}, \mathrm{o}}\right)$

The maximum cooling capacity, obtained if the entire refrigerant was evaporated, can be expressed as follows (see Fig. 2):

$\dot{\mathrm{Q}}_{\mathrm{ref}}=\dot{\mathrm{m}}_{\mathrm{ref}} \cdot\left(\mathrm{h}_{\mathrm{vE}}-\mathrm{h}_{\mathrm{iC}}\right)$

With $\dot{m}_{\text {ref }}$ obtained from a mass balance in the generator:

$\dot{m}_{\text {ref }}=\dot{m}_{\text {weak }}-\dot{m}_{\text {strong }}$

Eqs. (3) and (4) are compared in order to evaluate the quantity of evaporated refrigerant, and therefore the performance of evaporators-absorber combination. The evaluation of the cooling capacity of this facility in a previous work (GutiérrezUrueta et al., 2011) indicates evidence of refrigerant overflow on the evaporators. The fraction of refrigerant not evaporated,

Table 1 - Sensors used in the experimental test rig and the correspondent measurement uncertainty.

\begin{tabular}{lrll}
\hline Sensor type & Quantity & \multicolumn{1}{c}{ Range } & Uncertainty \\
\hline $\begin{array}{l}\text { Thermocouple } \\
\begin{array}{l}\text { Solution flow and } \\
\quad \text { density meter }\end{array}\end{array}$ & 30 & $5-110^{\circ} \mathrm{C}$ & $\pm(0.2 \mathrm{~K}-0.6 \mathrm{~K})$ \\
$\begin{array}{l}150-400 \mathrm{~kg} \mathrm{~h}^{-1} \\
\pm 0.5 \%\end{array}$ & \\
$\begin{array}{l}\text { Oil flowmeter } \\
\text { Cooling water }\end{array}$ & 1 & $1000-3600 \mathrm{l} \mathrm{h}^{-1}$ & $\pm 3.1 \%$ \\
$\quad$ flowmeters & 2 & $1200-1500 \mathrm{~kg} \mathrm{~h}^{-1} \pm 1.1 \%$ \\
$\begin{array}{l}\text { Recirculated solution } \\
\quad \text { flowmeters }\end{array}$ & 1 & $200-1000 \mathrm{~kg} \mathrm{~h}^{-1}$ & $\pm 1.3 \%$ \\
$\begin{array}{l}\text { Fan coil flowmeters } \\
\text { Pressure sensors }\end{array}$ & 2 & $400-600 \mathrm{~kg} \mathrm{~h}^{-1}$ & $\pm 0.7 \%$ \\
\hline
\end{tabular}

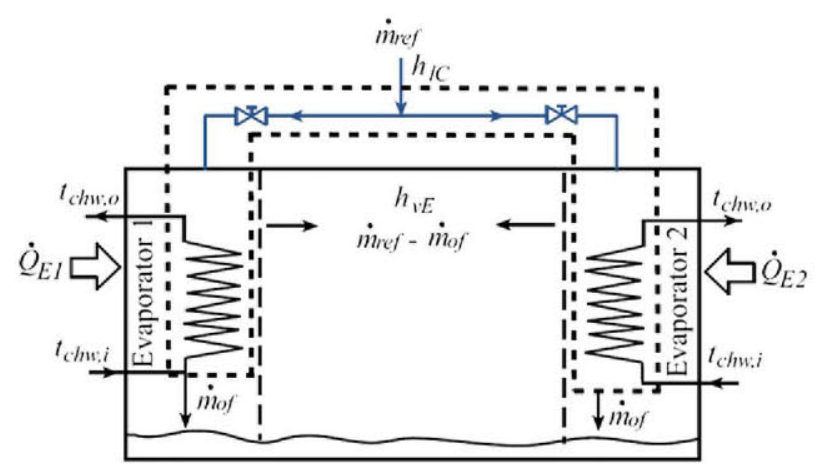

Fig. 2 - Control volume of evaporator (dashed lines).

named $\dot{m}_{\mathrm{of}}$, gives rise to a loss in refrigeration power, $\dot{\mathrm{Q}}_{\mathrm{of}}$, which may be obtained by subtracting Eqs. (4) and (3):

$\dot{\mathrm{Q}}_{\mathrm{of}}=\dot{\mathrm{m}}_{\mathrm{Of}} \cdot\left(\mathrm{h}_{\mathrm{VE}}-\mathrm{h}_{\mathrm{iE}}\right)$

Then, the energy balance in the evaporators, is expressed in a general way as:

$\dot{\mathrm{Q}}_{E}=\dot{m}_{\mathrm{ref}} \cdot\left(\mathrm{h}_{\mathrm{vE}}-\mathrm{h}_{\mathrm{ic}}\right)-\dot{\mathrm{Q}}_{\mathrm{of}}$

Using this expression, the evaporator overflow is then included in the analysis.

The solution heat exchanger efficiency $\eta_{\text {hex }}$ is defined as the ratio of the actual heat transfer to the maximum possible heat transfer for the given inlet conditions.

$\eta_{\text {hex }}=\frac{\dot{\mathrm{Q}}_{\text {hex }}}{\dot{\mathrm{Q}}_{\max }}$

With

$\dot{\mathrm{Q}}_{\text {hex }}=\left(\dot{m} c_{p}\right)_{\text {cold }}\left(\mathrm{T}_{\text {weak }, 0}-\mathrm{T}_{\text {weak }, \mathrm{i}}\right)=\left(\dot{m} c_{p}\right)_{\text {hot }}\left(\mathrm{T}_{\text {strong }, \mathrm{i}}-\mathrm{T}_{\text {strong, }, \mathrm{o}}\right)$

$\dot{Q}_{\max }=\left(\dot{m} c_{p}\right)_{\text {min }}\left(T_{\text {strong, } \mathrm{i}}-\mathrm{T}_{\text {weak,in }}\right)$

The logarithmic mean temperature difference is given as (Incropera, 1996):

$\Delta T_{i m}=\frac{\Delta T_{2}-\Delta T_{1}}{\ln \left(\Delta T_{2} / \Delta T_{1}\right)}$

For counterflow exchanger:

$\Delta \mathrm{T}_{1}=\mathrm{T}_{\text {hot, } \mathrm{i}}-\mathrm{T}_{\text {cold }, \mathrm{o}}$

$\Delta T_{2}=T_{\text {hot }, 0}-T_{\text {cold }, i}$

\section{Description of heat and mass transfer processes in components}

The main aspects of the heat and mass transfer processes in each component will be described next. Each aspect mentioned in this section is relevant to adapt the characteristic equations to this kind of machines.

The subscript " $\mathrm{I}$ " corresponds to ideal conditions, given by the average temperature of an ideal diabatic absorption process in equilibrium at the corresponding pressure.

For the later explanations, it is important to take into account that the use of a plate heat exchanger leads to apply a correction factor, $F$, if the number of plates is less than about 
50. $F$ is a function of the number of transfer units, NTU and of the number of plates. It takes into account that the two end plates of the heat exchanger transfer heat only by one of its sides, e.g. (Hewitt, 1998).

\subsection{Absorber}

Fig. 3 shows the control volume corresponding to the absorber.

Relevant aspects to be considered for the analysis of this component are listed in the following.

- In order to apply the characteristic equation methodology, the subcooler has been included inside the control volume since both heat and mass transfer processes have to be considered. Thus, interactions with the outside will include the same concepts existing in a diabatic absorber: heat transfer (both from the subcooler $\dot{Q}_{A}$ and losses to the environment $\dot{Q}_{\text {lossA }}$ ), as well as input and output enthalpy flows corresponding to strong solution, refrigerant, and weak solution. For machines equipped with a solution recirculation pump, the work done on the solution should be included in the control volume also.

- The half part of the solution heat exchanger, corresponding to the strong solution, has been included in the control volume of the absorber for compatibility with the methodology presented by Hellmann et al. (1998).

- Two adiabatic absorption processes, corresponding to strong and recirculated solution streams, are noted in Fig. 3

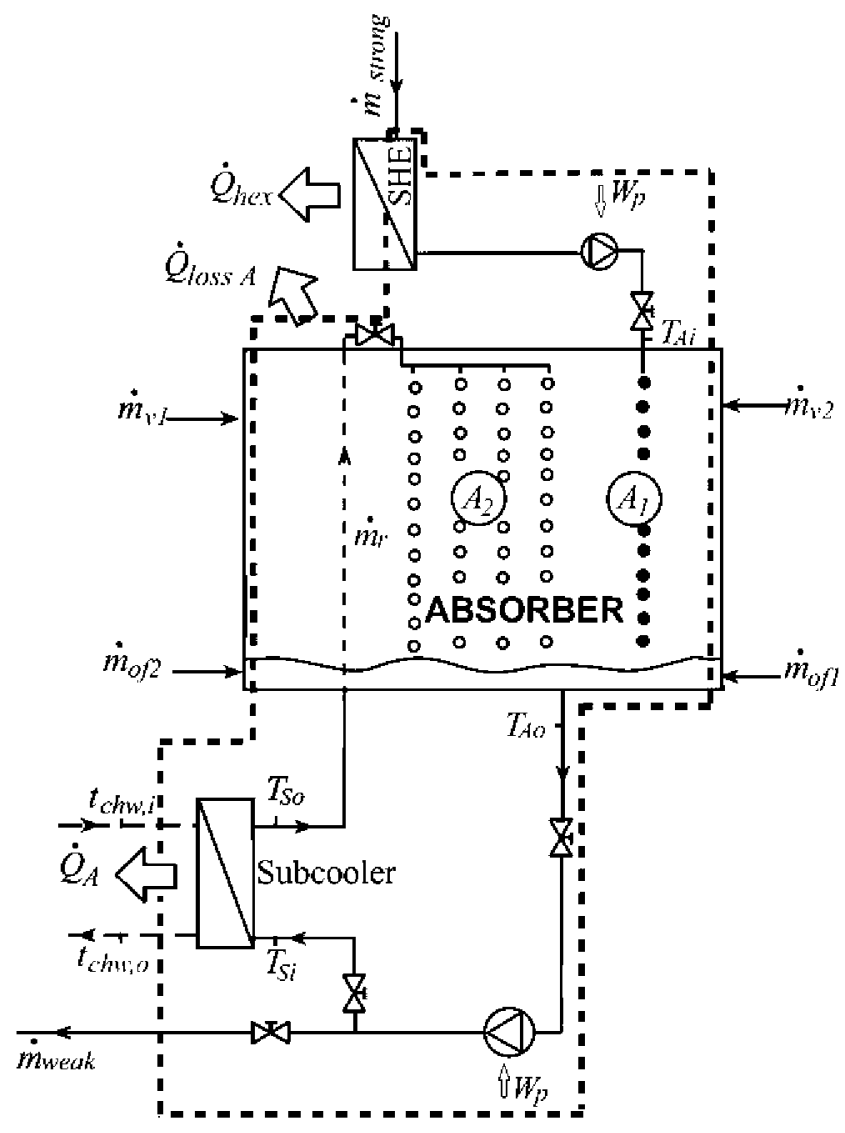

Fig. 3 - Control volume of absorber (dashed lines). as " $A_{1}$ " and " $A_{2}$ " respectively. The solution temperature corresponding to strong and recirculated streams, $T_{A i}$ and $T_{\text {So }}$ respectively, increases slightly while their concentration of salt decreases during the absorption processes $A_{1}$ and $A_{2}$. Finally, the solution from both $A_{1}$ and $A_{2}$ drops into the pool, where it mixes up, to be pumped to the subcooler and the generator in parallel.

- The value of solution temperature at absorber outlet, $T_{A 0}$, is considered as the same as that at subcooler entry, $T_{S i}$. This has been experimentally verified in this work and it means that the increase of temperature produced by the pump is insignificant and not perceptible with the instrumentation used.

- The mean value of the temperature of inlet and outlet of the weak solution temperature in the subcooler is named $T_{\text {Asub. }}$

- The heat transfer process takes place only in the subcooler. So, $T_{\text {Asub }}$ represents the internal fluid temperature $T_{A}$

$\mathrm{T}_{\mathrm{A}}=\frac{\mathrm{T}_{\mathrm{Ao}}+\mathrm{T}_{\mathrm{So}}}{2}=\mathrm{T}_{\text {Asub }}$

The difference between $T_{\text {Asub }}$ and $T_{A I}$ is named "absorber subcooling" $\left(\Delta T_{A}\right)$. It indicates the difference in mean solution temperature between the heat transfer process which takes place in a diabatic absorber (supposed to work under saturated conditions) and the one that occurs in an adiabatic absorber (where the heat transfer takes place far from saturated conditions).

- The heat transmission parameter $U A_{A}$ is obtained from the evaluation of the heat transfer rate using the logarithmic mean temperature difference and a correction factor $F_{A}$, Eq. (14).

$\dot{\mathrm{Q}}_{\mathrm{A}}=U \mathrm{~A}_{\mathrm{A}} \cdot \Delta \mathrm{T}_{\mathrm{ImA}} \cdot F_{\mathrm{A}}$

For the calculation of $\Delta T_{\text {ImA }}$ Eqs. (11) and (12) are applied, with the diluted solution and cooling water as hot and cold fluids, respectively, in the subcooler.

Thermal losses from the subcooler to the ambient are calculated as a part of $\dot{Q}_{\mathrm{Loss} A}$.

- External fluid temperature $t_{A}$ is defined as:

$t_{A}=\frac{t_{c w, o}+t_{c w, i}}{2}$

- The mass balance in the absorber is given by Eq. (16), according to Fig. 3 :

$\dot{m}_{\text {weak }}-\dot{m}_{\text {strong }}=\dot{m}_{\mathrm{v} 1}+\dot{m}_{\mathrm{v} 2}+\dot{m}_{\mathrm{of} 1}+\dot{m}_{\mathrm{of} 2}$

The subscripts 1 and 2 designate evaporator 1 and 2, respectively.

- The energy balance in the absorber is given by Eq. (17):

$$
\begin{aligned}
\dot{Q}_{A}= & \left(\dot{m}_{v 1}+\dot{m}_{v 2}\right) \cdot h_{v E}+\left(\dot{m}_{\text {of } 1}+\dot{m}_{\text {of } 2}\right) \cdot h_{\text {tE }}+\dot{m}_{\text {strong }} \cdot h_{\text {strong }} \\
& -\dot{m}_{\text {weak }} \cdot h_{\text {weak }}-\dot{Q}_{\text {hex }}-\dot{Q}_{\text {lossA }}
\end{aligned}
$$

Thermal loss to the environment $\dot{Q}_{\text {lossA }}$ is evaluated for the reason that the absorber shell exchanges heat with the 
environment, because the walls are in contact with the solution (which is hot after the absorption process). This is due to the splash from both the nozzle and the solution pool at the bottom. Mean values of temperatures measured at left and right side of the absorber is $41^{\circ} \mathrm{C}$. The ambient temperature ranges from $18^{\circ} \mathrm{C}$ to $27^{\circ} \mathrm{C}$ with a mean value of $23^{\circ} \mathrm{C}$. The loss is the order of $5 \%$ of the thermal power of the subcooler $\dot{Q}_{A}$, being this case favorable for the operation of the subcooler.

\subsection{Generator}

Fig. 4 shows the control volume corresponding to the generator.

Relevant aspects to be considered for the analysis of this component are listed in the following.

- The separator has been included inside the control volume since refrigerant separation continues in the route from the generator outlet to the separator outlet. Interactions with the outside are similar to those existing in a conventional diabatic absorption chiller: heat (both from the generator $\dot{Q}_{G}$ and losses to the environment $\dot{Q}_{\text {lossG }}$ ), input and output enthalpy flows corresponding to strong solution, refrigerant and weak solution.

- The half of the solution heat exchanger corresponding to the weak solution has been included in the control volume of the generator for compatibility with the methodology presented by Hellmann et al. (1998).

- The weak solution enters the generator at a sub-cooled condition, $T_{G i}$, depending on solution heat exchanger efficiency, Eq. (8).

- Due to the pressure drop in the generator channels by friction, acceleration and gravity, superheated condition of vapor is present in part of the plate heat exchanger generator.

- The mean value of inlet and outlet solution temperature in the generator $\left(T_{G i}\right.$ and $\left.T_{G o}\right)$ is named $T_{G s u b}$.

- The difference between $T_{G s u b}$ and $T_{G I}$ is named "generator subcooling". This quantity is smaller when the solution heat exchanger efficiency is higher.

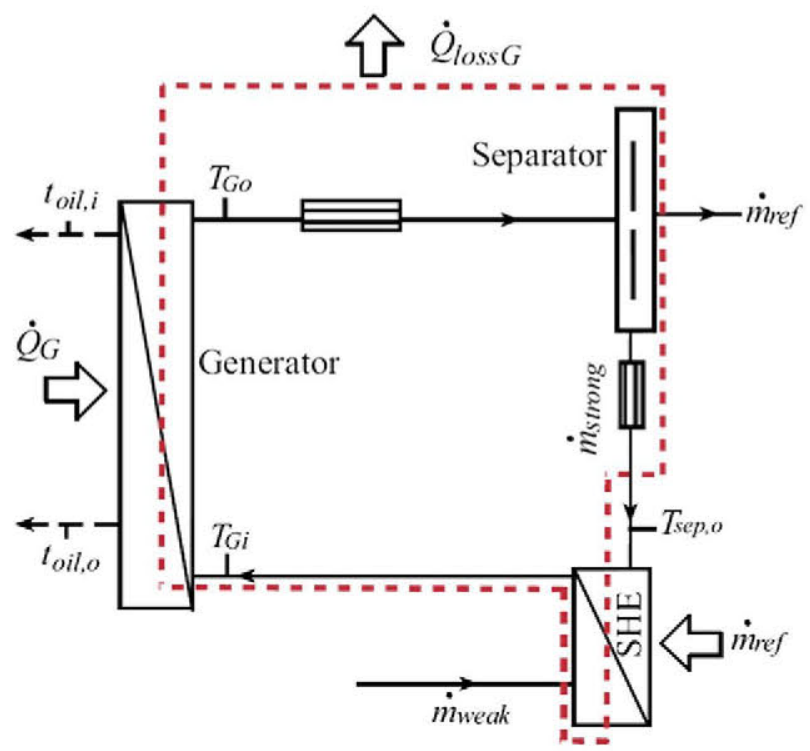

Fig. 4 - Control volume of generator (dashed lines).

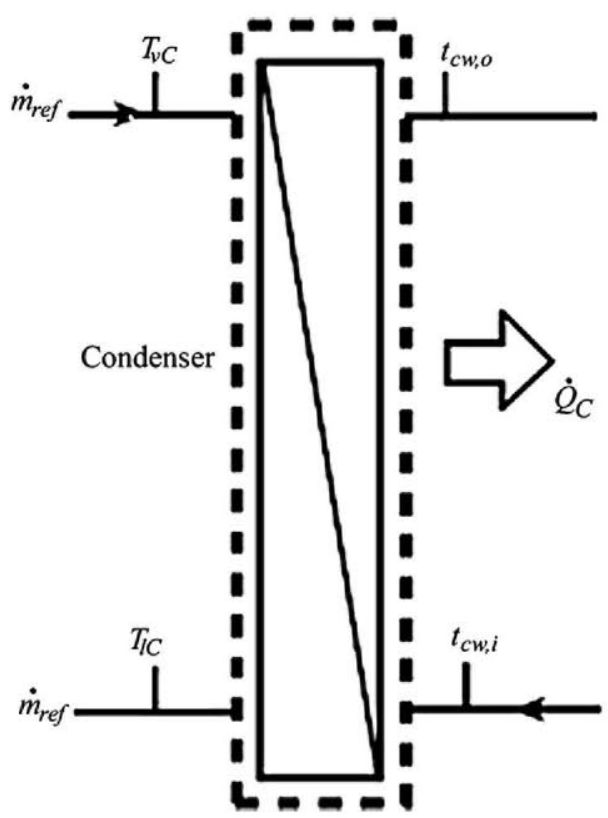

Fig. 5 - Control volume of condenser.

- The heat transmission $U A_{G}$ is obtained from the evaluation of the heat transfer rate using the logarithmic mean temperature difference and a correction factor $F_{\mathrm{G}}$, Eq. (18):

$\dot{\mathrm{Q}}_{\mathrm{G}}=U \mathrm{U}_{\mathrm{G}} \cdot \Delta \mathrm{T}_{\mathrm{imG}} \cdot \mathrm{F}_{\mathrm{G}}$.

The term $\Delta \mathrm{T}_{\text {ImG }}$ is estimated according to Eqs. (11) and (12), with the thermal oil and the solution as hot and cold fluids, respectively, in the generator.

- Downstream the exit of the heat exchanger, an adiabatic zone for vapor separation results in almost saturated condition, when the condenser pressure is attained in the droplets separator vessel.

- The heat transfer process takes place between $T_{G i}$ and $T_{G o}$ So, $T_{G s u b}$ represents the internal fluid temperature:

$\mathrm{T}_{\mathrm{G}}=\frac{\mathrm{T}_{\mathrm{Gi}}+\mathrm{T}_{\mathrm{Go}}}{2}=\mathrm{T}_{\mathrm{Gsub}}$

- External fluid temperature $t_{G}$ is defined as:

$t_{G}=\frac{t_{\mathrm{oil}, \mathrm{i}}+t_{\mathrm{oil}, \mathrm{o}}}{2}$

- The mass balance in the generator is given by Eq. (21), according to Fig. 5:

$\dot{m}_{\text {weak }}-\dot{m}_{\text {strong }}=\dot{m}_{\text {ref }}$

- The energy balance in the generator is expressed as:

$\dot{\mathrm{Q}}_{G}=\dot{\mathrm{m}}_{\mathrm{ref}} \cdot \mathrm{h}_{\mathrm{uG}}+\dot{\mathrm{m}}_{\text {strong }} \cdot \mathrm{h}_{\text {strong }}-\dot{\mathrm{m}}_{\text {weak }} \cdot \mathrm{h}_{\text {weak }}-\dot{\mathrm{Q}}_{\text {hex }}+\dot{\mathrm{Q}}_{\text {lossG }}$

A model of the heat transfer losses to the ambient evaluates the thermal losses to the environment $\dot{Q}_{\text {lossG }}$. They represent as average value $8 \%$ of the generation power $\dot{Q}_{G}$. 


\subsection{Evaporator}

Fig. 2 shows the control volume corresponding to the evaporator. As can be seen, the refrigerant distributor is considered as a component inside this control volume.

Relevant aspects to be considered are:

- The "overflow" $\dot{m}_{\text {of }}$, is considered in this evaporator scheme as a flow rate of non-evaporated refrigerant directly to the pool of the absorber as a consequence of an undesirable saturation of its evaporation capacity, leading to a loss of cooling capacity. In Kim and Infante Ferreira (2008) this situation is explained, as well as in other papers cited in this work.

- As both evaporators and the absorber are located in the same vessel, the refrigerant saturation temperature $T_{E}$ corresponds to absorber pressure $P_{A}$.

- Thermal input from the ambient to the evaporators can be considered negligible because of its location within the low pressure shell next to the absorber. Evaporators external walls are not wetted by the refrigerant, in contrast to the case of the absorber walls, wetted by the solution. Additionally, the evaporators are insulated from the absorber radiation heat through a double metallic flat plate, acting as a radiation shield. Radiation heat from the ambient to the evaporators can reach around $1.4 \%$ considering the ambient and evaporation temperatures (the mean value of evaporation temperature is $5^{\circ} \mathrm{C}$ ), so this loss can also be considered negligible for the purposes of this analysis.

- External fluid temperature $t_{E i}$ is defined for each evaporator as:

$t_{E i}=\frac{t_{\text {chwi }}+t_{\text {chwo }}}{2}$

In order to calculate $t_{\mathrm{E}}$, the mean value of $t_{\mathrm{E} 1}$ and $t_{\mathrm{E} 2}$ (corresponding to each evaporator) is used.

- The mass balance in the evaporators is given by Eq. (24), according to Fig. 2:

$\dot{m}_{\text {ref }}=\dot{m}_{\text {ref }_{1}}+\dot{m}_{\mathrm{ref}_{2}}$

The terms on the right side of Eq. (24) correspond to the distribution of refrigerant on both evaporators (GutiérrezUrueta et al., 2011).

In each evaporator there will be an evaporated (used) and not evaporated (overflow) part of refrigerant:

$\dot{m}_{\mathrm{ref}_{1}}=\dot{m}_{\mathrm{v}_{1}}+\dot{m}_{\mathrm{of}_{1}}$

$\dot{m}_{\mathrm{ref}_{2}}=m_{\mathrm{v}_{2}}+\dot{m}_{\mathrm{of}_{2}}$

where $\dot{m}_{\mathrm{v}_{1}}$ and $\dot{m}_{\mathrm{v} 2}$ designates the evaporated refrigerant.

Regarding the refrigerant mass flow rate that is not evaporated, an evaporator efficiency $\eta_{E}$ is expressed as:

$\eta_{E}=\frac{\dot{m}_{v}}{\dot{m}_{\text {ref }}}=\frac{\dot{m}_{\text {ref }}-\dot{m}_{\text {of }}}{\dot{m}_{\text {ref }}}=1-\frac{\dot{m}_{\text {of }}}{\dot{m}_{\text {ref }}}$

This is different from the heat exchanger efficiency. It depends on the absorber performance as well.
- The energy balance in the evaporators is given by Eq. (7).

- It is necessary to estimate a mean $U A_{E}$ for both evaporators. Values of $U A_{E}$ and $\Delta T_{I M E}$ are estimated by using the following equation system:

$\dot{\mathrm{Q}}_{E}=\dot{\mathrm{Q}}_{\mathrm{E} 1}+\dot{\mathrm{Q}}_{\mathrm{E} 2}=U \mathrm{~A}_{\mathrm{E} 1} \cdot \Delta \mathrm{T}_{\mathrm{im} 1}+U \mathrm{~A}_{\mathrm{E} 2} \cdot \Delta \mathrm{T}_{\mathrm{im} 2}$

$\dot{\mathrm{Q}}_{E}=U \mathrm{~A}_{E} \cdot \Delta \mathrm{T}_{\mathrm{imE}}$

From Eqs. (28) and (29):

$\Delta T_{i m E}=\Delta T_{i m 1} \frac{U A_{E 1}}{U A_{E}}+\Delta T_{i m 2} \frac{U A_{E 2}}{U A_{E}}$

If one of the evaporators does not receive any refrigerant due to a bad distribution, there is no heat exchange wet area $\left(A_{E i}=0\right)$, and the temperature difference $\Delta T_{i m E}$ is not defined. In those cases, $U A_{E}$ and $\Delta T_{i m E}$ would be corresponding to the one evaporator that receives the refrigerant.

As a first rough estimate it is assumed that $U A_{E}$ depends on the received refrigerant mass flow rate. Therefore, $\Delta T_{l m E}$ can be approximated as:

$\Delta T_{\text {imE }}=\Delta T_{\text {im } 1} \frac{m_{\text {ref1 }} \text { used }}{}+\Delta T_{\text {i } m 2} \frac{m_{\text {ref } 2_{\text {used }}}}{m_{\text {ref }}}$

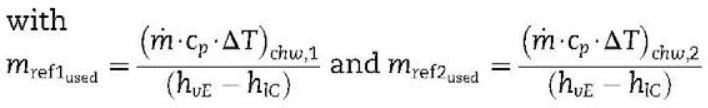

\subsection{Condenser}

Fig. 5 shows the control volume corresponding to the condenser.

- The energy balance in the condenser is expressed as:

$\mathrm{Q}_{\mathrm{C}}=\dot{m}_{\mathrm{ref}} \cdot\left(\mathrm{h}_{\mathrm{vC}}-\mathrm{h}_{\mathrm{iC}}\right)$

- Vapor is entering at the superheated condition.

- The refrigerant saturation temperature $T_{C}$ corresponds to the inlet condenser pressure $P_{C}$.

- The external fluid temperature $t_{C}$ is calculated as the mean value of inlet and outlet cooling water temperature:

$t_{C}=\frac{t_{c w i}+t_{c w o}}{2}$

- $U A_{C}$ is obtained from the evaluation of the heat transfer rate using the logarithmic mean temperature difference and a correction factor $F_{C}$, Eq. (35):

$\dot{\mathrm{Q}}_{\mathrm{C}}=U \mathrm{~A}_{C} \cdot \Delta \mathrm{T}_{\mathrm{ImC}} \cdot \mathrm{F}_{\mathrm{C}}$

For the estimation of $\Delta T_{l m C}$ Eqs. (11) and (12) are used, with the vapor and the cooling water as hot and cold fluids, respectively, in the condenser. In this case, the saturation temperature corresponding to the inlet condenser pressure $P_{C}$ is considered for the vapor side.

- Heat loss of the condenser can be neglected because of its temperature being near to the ambient $\left(24^{\circ} \mathrm{C}\right.$ as mean value). 
In order to illustrate the important ideas explained in the previous subsections, about the heat and mass transfer process in the components, Fig. 6 is presented. This scheme shows the solution cycle layout of the refrigerant vapor pressure against boiling temperature (lnP-1/T diagram), in a schematic way. In this paper, we understand the pressure to be the equilibrium pressure corresponding to the temperature and composition of the solution. It is not equal to the pressure which exists in the vessel. As already commented, the subscript "I" corresponds to ideal diabatic conditions. In this way, $T_{A I}$ and $T_{G I}$ represent the average value of the equilibrium temperatures of the strong and the weak solution in the absorber and the generator, respectively, at the corresponding pressures.

The adiabatic absorption processes, described in Section 4.1, " $A_{1}$ " and " $A_{2}$ " are represented in this figure. The inlet temperature of both strong and recirculated streams, $T_{A i}$ and $T_{S o}$ and their slight change, due to the absorption processes $A_{1}$ and $A_{2}$, are indicated by points " $a_{1}$ " and " $a_{2}$ ". Downstream, the solution from both $A_{1}$ and $A_{2}$ drops into the pool, where it mixes up to the conditions of temperature and concentration of point $S$. The point $S$, is nearer to the state of point $a_{2}$ than that of $a_{1}$ because the recirculated solution mass flow rate $\dot{m}_{r}$ is at least 1.5 times higher than the concentrated solution mass flow rate $\dot{m}_{\text {strong. The value of solution temperature at absorber }}$ outlet, $T_{A o}$, is considered as the same as that at subcooler entry, $\mathrm{T}_{\mathrm{Si}}$ (point S in Fig. 3). The heat transfer process (HT) occurs in the subcooler, with the mean solution temperature $T_{\text {Asub }}$ as well as the subcooling condition $\Delta T_{A}$ represented here.

The concentration measured at absorber outlet corresponds to $X_{\text {weak }}$. The ultimate equilibrium concentration $X_{\mathrm{eq}}$ corresponds to the concentration of the diluted solution when the solution is brought to equilibrium adiabatically. The estimation of $X_{\mathrm{eq}}$ is explained in previous works (GutiérrezUrueta et al., 2011; Ventas et al., 2010).

The generation process is represented as follows: the heat transfer process (HT) occurs between the inlet and outlet solution temperature $T_{G i}$ and $T_{G o}$. The condition of the solution at the generator exit is represented by point " $g$ " in Fig. 6 . The saturated condition and the "generator subcooling" are indicated as $T_{\text {sep,o }}$ and $\Delta T_{G}$, respectively.

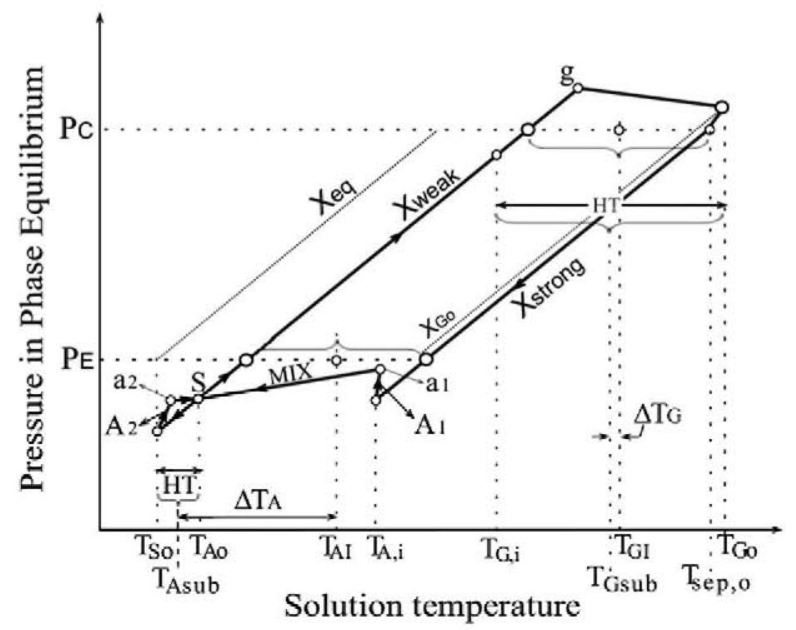

Fig. 6 - Schematic diagram of solution cycle in the adiabatic absorber on the $\ln \mathrm{P}-1 / \mathrm{T}$ diagram.

\section{Extension of the characteristic equation}

With the aim to implement a model which is able to predict the cooling capacity obtained for a wide range of operating conditions, the characteristic equation model for the described adiabatic absorption system has been investigated. It includes the specific features of both the design and the operation, which have been outlined in Section 4; mainly: plate heat exchangers, two evaporators, evaporator overflow and absorber subcooling. In the following, the procedure to adapt the characteristic equation to such features is presented.

Following the analysis developed by Hellmann et al. (1998), the energy fluxes corresponding to each component are expressed as a function of the difference between external and internal temperatures:

$\left|t_{i}-T_{i}\right| \quad ; i=E, C, A, G$.

As already explained, the feature of plate heat exchangers is considered by including the correction factor $F$.

$\dot{\mathrm{Q}}_{C}=U A_{C} \cdot z_{C} \cdot\left(T_{C}-t_{C}\right) \cdot F_{C}$
$\dot{Q}_{A}=U A_{A} \cdot z_{A} \cdot\left(T_{A}-t_{A}\right) \cdot F_{A}$
$\dot{Q}_{G}=U A_{G} \cdot z_{G} \cdot\left(t_{G}-T_{G}\right) \cdot F_{G}$
$\dot{Q}_{E}=U A_{E} \cdot z_{E} \cdot\left(T_{E}-t_{E}\right)$

The correction factor for the case of the evaporator is neglected, taking into account that the temperature of one of the fluids remains constant.

The terms $z_{i}$, Eq. (37) represents the correction needed because of considering $\left|t_{i}-T_{i}\right|$ instead of the logarithmic temperature difference $\Delta T_{l m, i}$ in the energy balance, as is done by Hellmann et al. (1998). Mean values of this factor were 0.9 for the generator, 0.8 for both condenser and subcooler and 0.7 for the case of the evaporator. The size of the correction depends on the different heat capacities $m \cdot C_{p}$ of the flows involved.

$z_{i}=\frac{\Delta T_{i m, i}}{\left|t_{i}-T_{i}\right|}$

It is a property of the working pairs that the difference between generator and absorber temperatures, $T_{G}-T_{A}$, is proportional to the difference of the condenser and evaporator temperature $T_{C}-T_{E}$. The proportionality factor, $B$, in very good approximation depends on the average solution concentration only:

$\mathrm{T}_{\mathrm{G}}-\mathrm{T}_{\mathrm{A}}-\mathrm{B} \cdot\left(\mathrm{T}_{\mathrm{C}}-\mathrm{T}_{\mathrm{E}}\right)=0$

The following analysis allows considering the absorber subcooling in the adaptation to the characteristic equation: The parameter B in Eq. (38) is the isostere slope in a Dühring diagram for an average solution concentration. $T_{G}$ and $T_{A}$ are generally considered as $T_{G I}$ and $T_{A I}$ (Fig. 3) respectively. But as previously explained, those are not the characteristics temperatures for the real heat transfer processes due to subcooling, which are $T_{G s u b}$ and $T_{\text {Asub }}$. Then, a definition of an apparent value of $B$, named $B_{\text {sub }}$ is necessary to satisfy Eq. (38):

$B_{\text {sub }}=\frac{T_{\text {Gsub }}-T_{\text {Asub }}}{T_{C}-T_{E}}$ 
This parameter can be related with $B$ :

$B_{\text {sub }}=B+\frac{\Delta T_{A}-\Delta T_{G}}{T_{C}-T_{E}}$

where, due to the characteristic subcooling process of the adiabatic absorbers, $\Delta T_{A}$ is the most relevant contribution.

For a diabatic absorber, the four temperatures $T_{G}, T_{A}, T_{C}$ and $T_{E}$ must be related to each other when applying the characteristic equation. In adiabatic absorbers, at least $\Delta T_{A}$ must also be specified. Additionally, no matter what type of absorber is used, $\eta_{\text {hex }}$ should be specified to calculate $\Delta T_{G}$.

Combining Eqs. (36) and (38):

$$
\begin{aligned}
& \frac{\dot{Q}_{G}}{U A_{G} \cdot z_{G} \cdot F_{G}}+\frac{\dot{Q}_{A}}{U A_{A} \cdot z_{A} \cdot F_{A}}+B_{\text {sub }} \cdot\left(\frac{\dot{Q}_{C}}{U A_{C} \cdot z_{C} \cdot F_{C}}+\frac{\dot{Q}_{E}}{U A_{E} \cdot z_{E}}\right) \\
& =t_{G}-t_{A}-B_{\text {sub }} \cdot\left(t_{C}-t_{E}\right)=\Delta \Delta t
\end{aligned}
$$

In order to express $\dot{Q}_{C}, \dot{Q}_{A}$ and $\dot{Q}_{G}$ as a function of $\dot{Q}_{E}$, the above equations are combined with the energy balance for the rest of the components (deduced previously in Section 4):As a result:

$$
\begin{aligned}
& \dot{Q}_{C}= \frac{\left(h_{v C}-h_{i C}\right)}{\left(h_{v E}-h_{i C}\right)} \cdot\left(\dot{Q}_{E}+\dot{Q}_{o f}\right)=K_{C} \cdot\left(\dot{Q}_{E}+\dot{Q}_{o f}\right) \\
& \dot{Q}_{A}=\frac{\left(h_{v E}-h_{\text {strongG }}\right)}{\left(h_{v E}-h_{i C}\right)} \cdot\left(\dot{Q}_{E}+\dot{Q}_{o f}\right)+\dot{m}_{\text {weak }} \cdot\left(h_{\text {strongG }}-h_{\text {weakA }}\right) \\
&-\dot{Q}_{\text {hex }}-\dot{Q}_{o f}-\dot{Q}_{\text {lossA }} \\
&= K_{A} \cdot \dot{Q}_{E}+\dot{Q}_{o f} \cdot\left(K_{A}-1\right)+\dot{Q}_{\text {loss }}-\dot{Q}_{\text {loss } A} \\
& \dot{Q}_{G}= \frac{\left(h_{v C}-h_{\text {strongG }}\right)}{\left(h_{v E}-h_{i C}\right)} \cdot\left(\dot{Q}_{E}+\dot{Q}_{o f}\right)+\dot{m}_{\text {weak }} \cdot\left(h_{\text {strongG }}-h_{\text {weakA }}\right) \\
&-\dot{Q}_{\text {hex }}+\dot{Q}_{\text {lossG }} \\
&= K_{G} \cdot\left(\dot{Q}_{E}+\dot{Q}_{\text {of }}\right)+\dot{Q}_{\text {loss }}+\dot{Q}_{\text {lossG }}
\end{aligned}
$$

Ratios of enthalpies differences, $K_{C}, K_{A}$ and $K_{G}$ are assumed to be constant, as a good approximation.

According to Hellmann et al. (1998), $\dot{Q}_{\text {loss }}$ is an equivalent for the solution heat exchanger internal loss, i.e. the heat required in the generator for heating, and rejected in the absorber for cooling the solution to the appropriate internal equilibrium temperature. Although this is an approximation (Albers and Ziegler, 2009) in this paper the assumption is preserved.

Combining Eqs (41) and (42) results in:

$\dot{\mathrm{Q}}_{E}=s \cdot \Delta \Delta \mathrm{t}-\alpha \cdot \mathrm{Q}_{\mathrm{loss}}-\beta \cdot \dot{\mathrm{Q}}_{\mathrm{of}}+\delta-\lambda$

$\Delta \Delta t$ is calculated according to Eq. (2) with using $B_{\text {sub }}$ instead of $B$.

The characteristic parameters $(s, \alpha, \beta, \delta$ and $\lambda)$ are defined as

$$
\begin{aligned}
& s=\frac{1}{\frac{K_{G}}{U A_{G} \cdot Z_{G} \cdot F_{G}}+\frac{K_{A}}{U A_{A} \cdot Z_{A} \cdot F_{A}}+B_{\text {sub }} \cdot\left(\frac{K_{C}}{U A_{C} \cdot Z_{C} \cdot F_{C}}+\frac{1}{U A_{E} \cdot Z_{E}}\right)} \\
& \alpha=s \cdot\left(\frac{1}{U A_{G} \cdot z_{G} \cdot F_{G}}+\frac{1}{U A_{A} \cdot z_{A} \cdot F_{A}}\right) \\
& \beta=s \cdot\left(\frac{K_{G}}{U A_{G} \cdot z_{G} \cdot F_{G}}+\frac{K_{A}-1}{U A_{A} \cdot Z_{A} \cdot F_{A}}+\frac{B_{\text {sub }} \cdot K_{C}}{U A_{C} \cdot Z_{C} \cdot F_{C}}\right) \\
& \delta=s \cdot\left(\frac{Q_{\text {loss }}}{U A_{A} \cdot Z_{A} \cdot F_{A}}\right) \\
& \lambda=s \cdot\left(\frac{Q_{\text {lossG }}}{U A_{G} \cdot Z_{G} \cdot F_{G}}\right)
\end{aligned}
$$

The parameters $\beta, \delta$ and $\lambda$ are new terms added to the original characteristic equation. Such parameters appear because of the effects of both overflow and thermal losses in the absorber and generator, respectively, giving a higher precision in the prediction of performance.

Section 6 shows results of this extension of the characteristic equation model for all the experimental points obtained.

\section{Results and discussion}

\subsection{Extended characteristic equation}

Fig. 7b illustrates the comparison of the experimental and predicted cooling capacity $\dot{Q}_{E}$, obtained by applying the characteristic equation model using " $B$ " instead of " $B_{\text {sub }}$ " and $\beta, \delta$ and $\lambda$. The need of including the subcooling effects on $B$ is revealed by the comparison with Fig. 7a. In this case $\dot{Q}_{E}$ was obtained through the extended characteristic equation. The parameter $B_{\text {sub }}$ varies from 1.16 to 1.65. Regarding Eq. (40), the contribution of the term $\Delta T_{A}$ ranges from $10 \mathrm{~K}$ to $28 \mathrm{~K}$, with an average value of $20 \mathrm{~K}$ in the operating conditions tested. The term $\Delta T_{G}$ ranges from $2 \mathrm{~K}$ to $5.2 \mathrm{~K}$ with an average value of
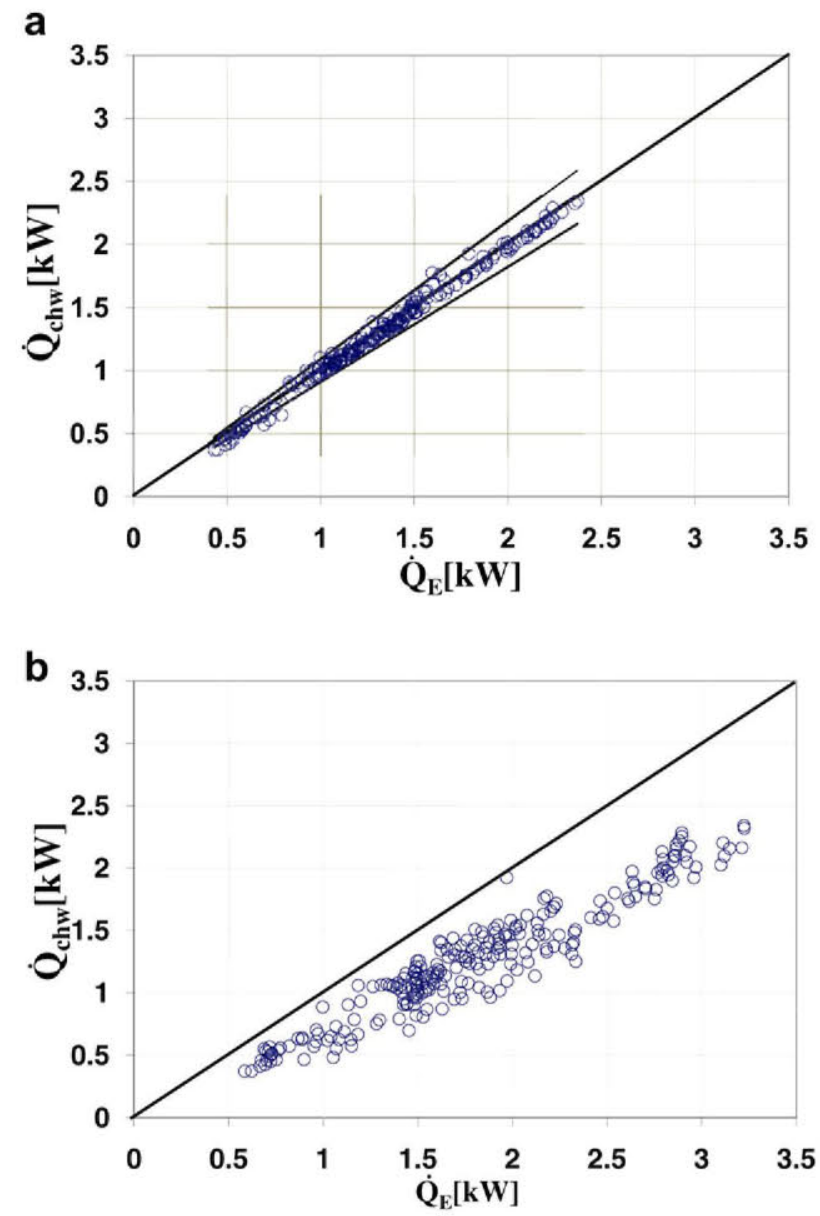

Fig. 7 - Comparison of cooling capacity obtained trough extended characteristic equation, $\dot{Q}_{E}$, with experimental data $\dot{Q}_{c h w}$. (a) Results using $B_{\text {sub. The }} 95 \%$ confidence interval is shown. (b) Results using $B$. 
$3.5 \mathrm{~K}$, for a solution heat exchanger efficiency $\eta_{\text {hex }}=0.65, \mathrm{Eq}$. (8).The ranges of the correction factors $F_{A}, F_{G}$ and $F_{C}$ are: $0.93<F_{A}<0.97 ; 0.95<F_{G}<0.98$ and $0.82<F_{C}<0.83$. The observed agreement is good and deviations are in the range of the overall uncertainty in the measured parameters.

The effect of overflow on $\dot{Q}_{E}$ is presented in Fig. 8a. Obviously, as the fraction of non-evaporated refrigerant $\dot{m}_{\mathrm{of}}$ increases, $\dot{Q}_{E}$ decreases. The data corresponding to low and high overflow mass flow rate $\left(\dot{m}_{\mathrm{of}}<0.4\right.$ and $\left.\dot{m}_{\mathrm{of}}>1.2\right)$ is shown separately in the characteristic plot (Fig. 8b) for different solution mass flow rates. The ideal linear behavior of the data when the overflow is almost null is manifested.

\subsection{Cooling capacity}

The influence of $\Delta \Delta t$ on cooling capacity was analyzed for 280 experimental points, using $B_{\text {sub. Fig. }} 9$ shows the performance parameters obtained for two methods of dispersing the solution: a spray producing droplets and one producing fan sheets, as a function of the characteristic temperature difference $\Delta \Delta t$ with a fixed recirculation ratio $R R$ and weak solution mass flow rate $\dot{m}_{\text {weak }}$. It can be noticed that $\dot{Q}_{\text {ref }}$ (maximum cooling capacity) representing the design conditions follows a linear
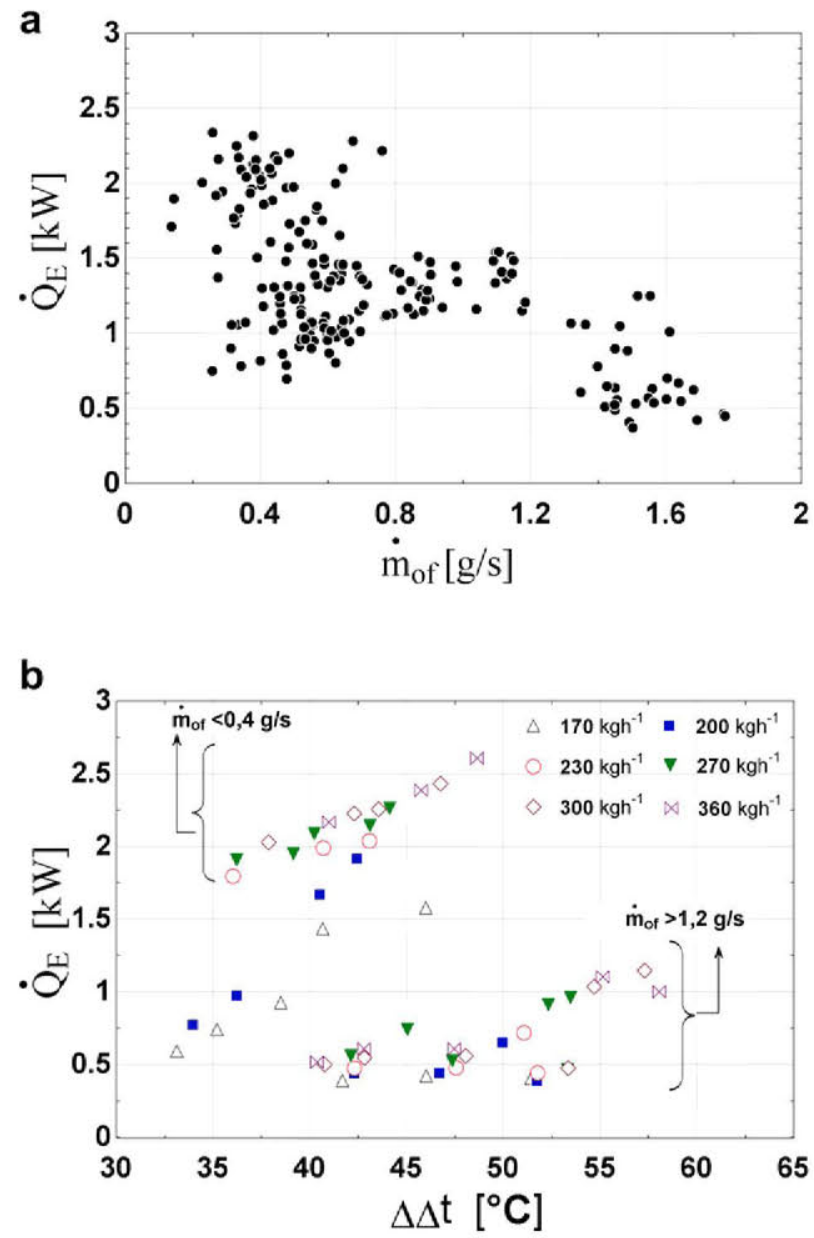

Fig. 8 - (a) Effect of evaporators' efficiency over the characteristic parameters. (b) Characteristics plot corresponding to low and high overflow mass flow rate for different solution mass flow rates.
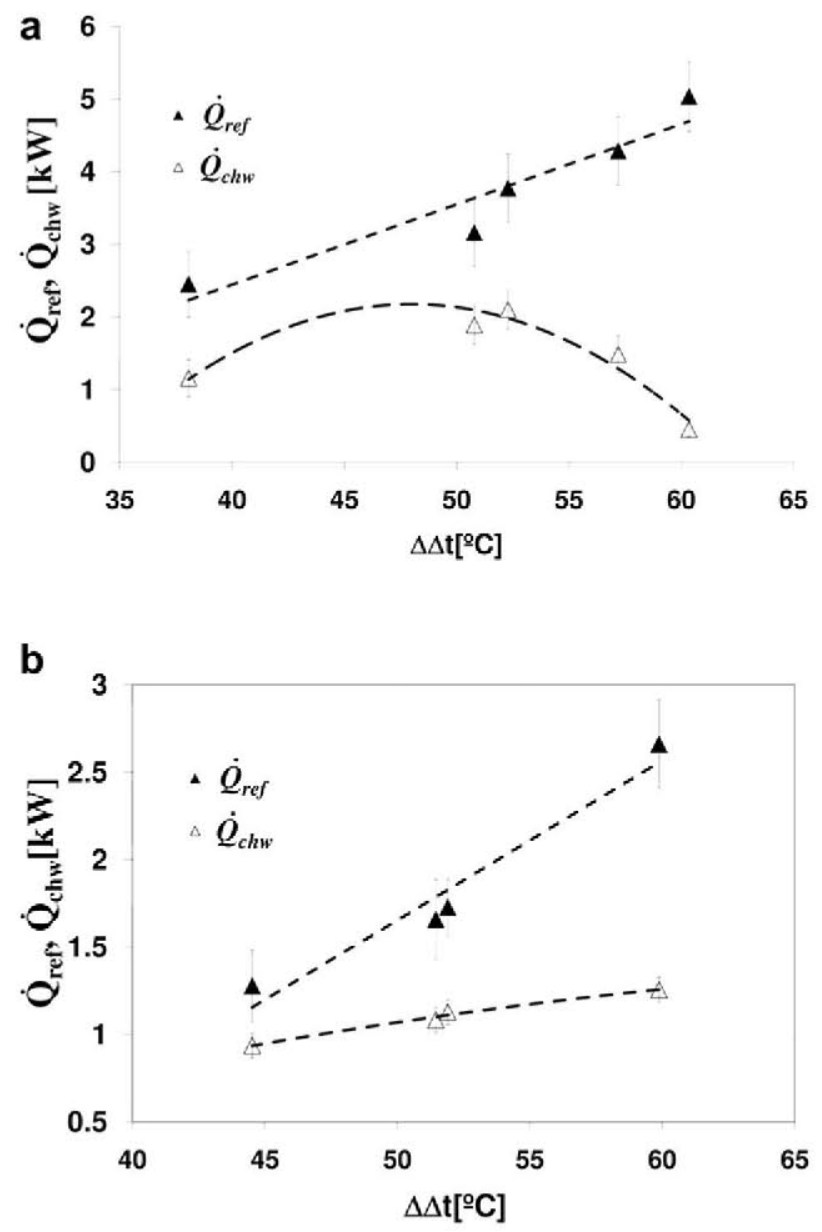

Fig. 9 - Cooling capacity vs. $\Delta \Delta$ t. (a) Falling drops, $\dot{m}_{\text {weak }}$ is fixed at $300 \mathrm{~kg} \mathrm{~h}^{-1}$ and $R R=1.9$. (b) Fan sheets, $\dot{m}_{\text {weak }}$ is fixed at $120 \mathrm{~kg} \mathrm{~h}^{-1}$ and $R R=2.9$.

behavior, increasing when $\Delta \Delta t$ increases. However, the measured actual cooling capacity is much less (Fig. 9b) and even drops off (Fig. 9a). This behavior was found similar for all values of $\dot{m}_{\text {weak }}$. The average loss in cooling capacity due to overflow obtained corresponds to $52 \%$ of $\dot{Q}_{\text {ref }}$, which is manifested by the difference between $\dot{Q}_{\text {ref }}$ and $\dot{Q}_{c h w}$. Previous works have also reported a similar behavior in the evaporator of some conventional absorption chillers such as Yazaki WFC10, e.g. (Albers and Ziegler, 2005). The evaporators performance loss causes the maximum value observed for $\dot{Q}_{\text {chw }}$ in Fig. 9. This is also discussed in Kim and Infante Ferreira (2008). The comparison of the two types of absorbers (liquid sheets and droplets as solution spreading mechanisms in the absorber) is discussed in a previous work (Gutiérrez-Urueta et al., 2011), which shows that the liquid sheet has shown better performance parameters than the droplet spray for this working solution. But it can be observed that independently of the configuration used for spreading the solution inside the absorber, the loss in cooling capacity due to the effect of the overflow is observed in both cases.

Due to its simplicity, this extended characteristic equation method can be applied in cases where the adiabatic absorption system needs to be simulated as part of a complete 
integrated system. The use of two algebraic equations to predict the part load behavior of the chiller eludes the necessity for extensive numerical simulation of the internal thermodynamic cycle, as commented by Hellmann et al. (1998).

The present work implements an extended model of the characteristic equation to adiabatic absorbers with subcooling, adding typical off-design operation parameters like the overflow. It also adds the observed effect of an excess of temperature, superheating, in boiling generators, such as plate heat exchangers based ones. This makes the model useful for purposes of operation and control of these machines and for avoiding not desirable or less efficient operating conditions.

\section{Conclusions}

The characteristic equation has been adapted to a specially designed single effect $\mathrm{H}_{2} \mathrm{O}-\mathrm{LiBr}$ absorption chiller and it has been applied for the experimental evaluation of the chiller performances.

An extension of the characteristic equation method to adiabatic absorption has been developed. For adiabatic absorbers, a subcooling temperature must be specified.

The effect of evaporator overflow has also been characterized. Its influence on the cooling capacity $\dot{Q}_{E}$ has been included in the extended characteristic equation.

Taking into account the particular design and operational features, a good agreement between experimental cooling capacity and those obtained through the extended characteristic equation has been achieved at off-design operation. This allows its use for simulation and control purposes.

\section{Acknowledgments}

The financial support of this study by the Ministry of Education, Science and Technology through CLIMABCAR project DPI 2003-01567, PIVEROT project CCG08-UC3M/ENE-4432 and MINICOM project (FIT 0204-2004-68 and FIT 020100-2003-233), is greatly appreciated. The authors express their gratitude to the technicians of Universidad Carlos III de Madrid Mr. Manuel Santos and Mr. Carlos Cobos for their invaluable help in this work.

\section{R E F E R E N G E S}

Albers, J., Ziegler, F., 2005. Improved control strategies for solar assisted cooling systems with absorption chillers using a thermosyphon generator. In: Proceedings of the Int. Solar Air Cond., October 16-17, Kloster Banz, Germany.

Albers, J., Ziegler, F., 2009. Influence of internal irreversibilities on the characteristic equation of absorption chillers. In: Proceedings of Heat Powered Cycle Conf. 2009, Berlin, p. 420.
Arzoz, D., Rodriguez, P., Izquierdo, M., 2005. Experimental study on the adiabatic absorption of water vapour into $\mathrm{H}_{2} \mathrm{O}-\mathrm{LiBr}$ solutions. Appl. Therm. Eng. 25 (5-6), 797-811.

Elperin, T., Fominykh, A., Orenbakh, Z., 2007. Coupled heat and mass transfer during nonisothermal absorption by falling droplet with internal circulation. Int. J. Refrigeration 30 (2), 274-281.

Florides, G.A., Kalogirou, S.A., Tassou, S.A., Wrobel, L.C., 2003. Design and construction of a $\mathrm{LiBr}$-water absorption machine. Energy Convers. Manage. 44 (15), 2483-2508.

Gutiérrez-Urueta, G., Rodríguez, P., Venegas, M., Ziegler, F., Rodríguez-Hidalgo, M.C., 2011. Experimental performances of a Libr-water absorption facility equiped with adiabatic absorber. Int. J. Refrigeration 334 (8), 1749-1759.

Hellmann, H.M., Schweigler, C., Ziegler, F., 1998. A simple method for modelling the operating characteristics of absorption chillers. In: Proceedings of Thermodynamics Heat and Mass Transfers of Refrigeration Machines and Heat Pumps Seminar EUROTHERM N ${ }^{\circ}$ 59, France, pp. 219-226.

Hewitt, G.F., 1998. Heat Exchanger Design Handbook (V.1). Begell House, New York.

Incropera, F.P., DeWitt, D.P., 1996. Fundamentals of Heat and Mass Transfer, fourth ed. John Wiley and Sons, New York.

Joudi, K.A., Lafta, A.H., 2001. Simulation of a simple absorption refrigeration system. Energy Convers. Manage. 42 (13), 1575-1605.

Kim, D.S., Infante Ferreira, C.A., 2008. Analytic modelling of steady state single-effect absorption cycles. Int. J. Refrigeration 31, 1012-1020.

Kühn, A., Ziegler, F., Operational results of a $10 \mathrm{~kW}$ absorption chiller and adaptation to the characteristic equation. In: Proceedings of the Int. Conf. Sol. Air Cond. 6-7, October 2005, Bad Staffelstein, Germany.

Ryan, W.A., 1994. Water absorption in an adiabatic spray of aqueous lithium bromide solution. In: Proceedings of the Int. Absorption Heat Pump Conf., AES, vol. 31. ASME, New York, pp. 155-162.

Ryan, W.A., Ruiz, F., Wurm, J., 1995. Model development and verification of spray absorption for gas driven cooling systems. In: Proceedings of the Int. Gas Research Conf., Cannes, pp. 1483-1493.

Summerer, F., Riesch, P., Ziegler, F., Alefeld, G., 1997. Hydroxide absorption heat pumps with spray absorber. ASHRAE Tech. Data Bull. 12 (1), 50-57.

Venegas, M., Izquierdo, M., Rodríguez, P., Lecuona, A., 2004. Heat and mass transfer during absorption of ammonia vapour by $\mathrm{LiNO}_{3}-\mathrm{NH}_{3}$ solution droplets. Int. J. Heat Mass Transfer 47 (12-13), 2653-2667.

Venegas, M., Rodríguez, P., Lecuona, A., Izquierdo, M., 2005. Spray absorbers in absorption systems using lithium nitrate-ammonia solution. Int. J. Refrigeration 28 (4), 554-564.

Ventas, R., Lecuona, A., Legrand, M., Rodríguez-Hidalgo, M.C., 2010. On the recirculation of ammonia-lithium nitrate in adiabatic absorbers for chillers. Appl. Therm. Eng. 30 (17-18), 2770-2777.

Wang, L., Chen, G.M., Wang, Q., Zhong, M., 2007. Thermodynamic performance analysis of gas-fired air-cooled adiabatic absorption refrigeration systems. Appl. Therm. Eng. 27 (8-9), 1642-1652.

Warnakulasuriya, F.S.K., Worek, W.M., 2006. Adiabatic water absorption properties of an aqueous absorbent at very low pressures in a spray absorber. Int. J. Heat Mass Transfer 49 (9-10), 1592-1602. 